\title{
Digital Geometry Processing with Topological Guarantees
}

\author{
Dinesh Manocha \\ Department of Computer Science \\ University of North Carolina at Chapel Hill \\ dmecs. unc . edu \\ http://gamma.cs.unc.edu/recons
}

\section{Introduction}

We describe novel approaches to compute reliable solutions for many non-linear geometric problems that arise in geometric modeling, computer graphics and robotics. Specifically, we focus on problems that can be formulated as surface extraction problems. These include Boolean operations and Minkowski sums of polyhedral or higher models as well as reliable polygonization of general implicit surfaces. All these problems reduce to computing a topology preserving isosurface from a volumetric grid, i.e. the zero set of a scalar field. A common way of representing a scalar field is to discretize the continuous scalar field into discrete samples - to compute the value of the scalar field at the vertices of a volumetric grid. We refer to this step as a sampling of the scalar field. The grid is an approximate representation of the scalar field; the accuracy of the approximate representation depends on the rate of sampling - the resolution of the grid. An explicit boundary representation of the implicit surface can be obtained by extracting the zero-level isosurface using Marching Cubes or any of its variants. We refer to these isosurface extraction algorithms collectively as MC-like algorithms. The output of an MC-like algorithm is an approximation - usually a polygonal approximation - of the implicit surface. We refer to this step as reconstruction of the implicit surface.

Our goal is to exploit the desirable properties of implicit surface representations for geometric computations such as Boolean operations (i.e. union, intersection and difference), Minkowski sum computation, simplification, configuration space boundary computation and remeshing. In each case, we wish to obtain an accurate polygonal approximation of the boundary of the final solid. Let E denote this boundary. We represent E implicitly - as an isosurface of a scalar field. This scalar field is obtained by performing minimum/maximum operations over the scalar fields defined for the primitives. At a broad level, our approach performs three main steps.

1. Sampling: Generate a volumetric grid and compute a scalar field (e.g, a signed distance field) at its corner grid points.

2. Geometric operation: For each geometric operation (union or intersection), perform an analogous operation (e.g., $\min / \max$ ) on the scalar fields of the primitives. At the end of this step, the scalar values at the grid points define a sampled scalar field for $\mathrm{E}$. 
3. Reconstruction: Perform isosurface extraction using an MC-like algorithm to obtain a topologically accurate polygonal approximation E. In fact, we guarantee that the reconstructed surface is homeomorphic to the exact surface without explicitly computing the exact surface representation.

Preserving topology is also important in many applications. In CAD, topological features such as tunnels often correspond to distinguishing characteristics of the model. The geometric models used to represent the organs in medical datasets often consist of handles. Retaining these topological features can be necessary in order to preserve the anatomical structure of the organ, which can be crucial for visualization and analysis. Apart from capturing important features present in E, guaranteeing topology is important for another reason. An algorithm that preserves topology avoids the introduction of extraneous topology; its output does not have unwanted additional components or handles.

We present a novel approach to compute a topology preserving isosurface using an MC-like algorithm for geometry processing applications. We present conservative sampling criteria such that if every cell in the volumetric grid satisfies the criteria, then the extracted isosurface will have the same topology as the exact isosurface. We present an adaptive subdivision algorithm to generate a volumetric grid such that every grid cell satisfies the sampling criteria. We present efficient computational techniques to verify the sampling criteria during grid generation. Our algorithm can easily perform these computations on polyhedra, algebraic or parametric primitives and their Boolean com- binations. Furthermore, we extend the adaptive subdivision algorithm to also bound the Hausdorff distance between the exact isosurface and the extracted isosurface. This ensures that the extracted isosurface is geometrically close to the exact isosurface. We have used our algorithm to perform accurate boundary evaluation of Boolean combinations of polyhedral and low degree algebraic primitives, model simplification, and remeshing of complex models. In each case, we compute a topology preserving polygonal app- roximation of the boundary of the final solid. The running time of our algorithm varies between a few seconds for simple models consisting of thousands of triangles and tens of seconds on complex primitives represented using hundreds of thousands of triangles on a desktop PC.

Some of the main benefits of our approach include:

- Conservative sampling criteria for the volumetric grid such that the topology of the isosurface is preserved $[1,4]$.

- An efficient adaptive subdivision algorithm to generate an octree satisfying the sampling criteria [1].

- Efficient and accurate algorithms for boundary evaluation of solids defined by Boolean operations. A fast algorithm to compute topology preserving simplification and remeshing of a complex polygonal model [1].

- An accurate algorithm to compute the Minkowski sum of 3D polyhedral models [2].

- A reliable algorithm to polygonize implicit surfaces based on star-shaped decomposition [6].

- Accurate algorithm to compute the free-space of low degree of freedom rigid and articulate models among polygonal obstacles [5].

- Exact motion planning for low degree of freedom robots, including first practical and reliable algorithms for path non-existence $[3,4]$. 
We will give an overview of our approach and demonstrate the applications. Overall, this approach provides a powerful framework to perform topologically reliable computations on a discretized geometric grid for a variety of applications.

\section{Acknowledgements}

This is joint work with my current and former students and postdocs including Gokul Varadhan, Shankar Krishnan, Young Kim, TVN Sriram and Liangjun Zhang. This work was supported in part by grants from National Science Foundation, Army Research Office, Office of Naval Research, DARPA, RDECOM and Intel.

\section{References}

1. Varadhan, G., Krishnan, S., Sriram, T.V.N., Manocha, D.: Topology Preserving Surface Extraction using Adaptive Subdivision. In: Eurographics Symposium on Geometry Processing (2004)

2. Varadhan, G., Manocha, D.: Accurate Minkowski Sum Approximation of Polyhedral Surfaces. In: Proceedings of Pacific Graphics (2004)

3. Varadhan, G., Krishnan, S., Sriram, T.V.N., Manocha, D.: A Simple Algorithm for Motion Planning of Translating Polyhedral Robots. In: Workshop on Algorithmic Foundations of Robotics (2004)

4. Varadhan, G., Manocha, D.: Star-shaped Roadmaps - A Deterministic Sampling Approach for Complete Motion Planning. In: Proceedings of Robotics: Science and Systems (2005)

5. Varadhan, G., Kim, Y., Krishnan, S., Manocha, D.: Topology Preserving Free Configuration Space Approximation. In: Proceedings of IEEE International Conference on Robotics and Automation (2006)

6. Varadhan, G., Krishnan, S., Zhang, L., Manocha, D.: Reliable Implicit Surface Polygonization using Visibility Mapping. In: Proceedings of Eurographics Symposium on Geometry Processing (2006) 